\title{
Bcl-2-associated athanogene 3 protects the heart from ischemia/reperfusion injury
}

Feifei Su, ${ }^{1,2}$ Valerie D. Myers, ${ }^{1}$ Tijana Knezevic, ${ }^{3}$ JuFang Wang, ${ }^{4}$ Erhe Gao, ${ }^{4}$ Muniswamy Madesh, ${ }^{4}$ Farzaneh G. Tahrir, ${ }^{3}$ Manish K. Gupta, ${ }^{3}$ Jennifer Gordon, ${ }^{3}$ Joseph Rabinowitz, ${ }^{4}$ Frederick V. Ramsey, ${ }^{5}$ Douglas C. Tilley, ${ }^{4}$ Kamel Khalili, ${ }^{3}$ Joseph Y. Cheung, ${ }^{1,4}$ and Arthur M. Feldman ${ }^{1}$

'Department of Medicine, Lewis Katz School of Medicine at Temple University, Philadelphia, Pennsylvania USA ${ }^{2}$ Department of Cardiology, Tangdu Hospital, Fourth Military Medical University, Xi'an, China. ${ }^{3}$ Department of Neuroscience, ${ }^{4}$ Center for Translational Medicine, and ${ }^{5}$ Department of Clinical Sciences, Lewis Katz School of Medicine at Temple University, Philadelphia, Pennsylvania USA.

Bcl-2-associated athanogene 3 (BAC3) is an evolutionarily conserved protein expressed at high levels in the heart and the vasculature and in many cancers. While altered BAC3 expression has been associated with cardiac dysfunction, its role in ischemia/reperfusion (I/R) is unknown. To test the hypothesis that BAC3 protects the heart from reperfusion injury, in vivo cardiac function was measured in hearts infected with either recombinant adeno-associated virus serotype 9-expressing (rAAV9-expressing) BAC3 or GFP and subjected to I/R. To elucidate molecular mechanisms by which BAC3 protects against I/R injury, neonatal mouse ventricular cardiomyocytes (NMVCs) in which BAC3 levels were modified by adenovirus expressing (Ad-expressing) BAC3 or siBAC3 were exposed to hypoxia/reoxygenation (H/R). H/R significantly reduced NMVC BAG3 levels, which were associated with enhanced expression of apoptosis markers, decreased expression of autophagy markers, and reduced autophagy flux. The deleterious effects of $H / R$ on apoptosis and autophagy were recapitulated by knockdown of BAC3 with Ad-siBAC3 and were rescued by Ad-BAC3. In vivo, treatment of mice with rAAV9-BAC3 prior to I/R significantly decreased infarct size and improved left ventricular function when compared with mice receiving rAAV9-GFP and improved markers of autophagy and apoptosis. These findings suggest that BAC3 may provide a therapeutic target in patients undergoing reperfusion after myocardial infarction.

Conflict of interest: The authors have declared that no conflict of interest exists.

Submitted: September 26, 2016 Accepted: October 20, 2016 Published: November 17, 2016

Reference information: JCI Insight. 2016;1(19):e90931. doi:10.1172/jci.insight.90931.

\section{Introduction}

Bcl-2-associated athanogene 3 (BAG3) is a 575-amino acid protein that is highly conserved in nature (1). First identified by its ability to bind Bcl-2, BAG3 is expressed most abundantly in the heart and skeletal muscle and in many cancers. BAG3 serves as a cochaperone with members of the heat shock family of proteins to regulate protein quality control, interacts with Bcl-2 to inhibit apoptosis, and maintains the structural integrity of the sarcomere by linking filamen with the Z-disc through binding with the actin capping protein $\beta-1$ (CapZ $\beta 1)$ (2).

Recent studies have demonstrated that BAG3 plays a pivotal role in maintaining cardiac homeostasis: (a) homozygous deletion of BAG3 in mice led to severe LV dysfunction, myofibril disorganization, and death by 4 weeks of age (3); (b) a single allele mutation in children was associated with progressive limb and axial muscle weakness, severe respiratory insufficiency, and cardiomyopathy $(4,5)$, whereas deletions in BAG3 have been associated with heart failure with reduced ejection fraction (HFrEF) independent of peripheral muscle weakness or neurologic complications $(6,7)$; (c) BAG3 levels were reduced in mice and pigs with HFrEF secondary to a LAD occlusion and in patients with end-stage HFrEF (6); and (d) knockdown of BAG3 in neonatal myocytes led to myofibrillar disarray when the cells were stretched (8). However, in adult myocytes, BAG3 localized at the sarcolemma and t-tubules, where it modulates myocyte contraction and action potential duration through specific interaction with the $\beta 1$-adrenergic receptor and L-type $\mathrm{Ca}^{2+}$ channel (9). Furthermore, overexpression of BAG3 using an adeno-associated virus serotype 9 (rAAV9-BAG3) restored left ventricular (LV) function in mice with diminished LV function secondary to a myocardial infarction (10). 
A common feature in patients with single nucleotide polymorphisms in BAG3 and myofibrillar myopathy is abnormalities in mitochondrial structure (11). Consistent with this pathological observation, we recently found that BAG3 promoted the clearance of damaged mitochondria through the autophagy-lysosome pathway and through direct interactions with mitochondria (12). By contrast, BAG3 knockdown significantly reduced autophagy flux, leading to the accumulation of damaged mitochondria and an increase in apoptosis (12). It is well known that mitochondrial dysfunction and damage are central to the pathophysiology of ischemia/reperfusion (I/R) injury, as the inability to eliminate damaged mitochondria leads to increased production of ROS and, ultimately, to cell death $(13,14)$. Therefore, we hypothesized that BAG3 might play a role in I/R injury. To test this hypothesis, we utilized both an in vitro model of hypoxia/ reoxygenation (H/R) in neonatal mouse ventricular cardiomyocytes (NMVCs) and an in vivo model of $\mathrm{I} / \mathrm{R}$ in adult mice. We report that both $\mathrm{H} / \mathrm{R}$ and $\mathrm{I} / \mathrm{R}$ are associated with decreased levels of BAG3 and that overexpression of BAG3 in mice prior to I/R significantly reduced infarct size and improved LV function.

\section{Results}

$H / R$ decreases BAG3 levels in NMVCs. BAG3 levels were significantly decreased in NMVCs after H/R (Figure $1, \mathrm{~A}$ and $\mathrm{B} ; P<0.01$ ) when compared with normoxic controls. To explore potential signaling pathways by which reduced BAG3 levels after H/R might influence cell injury, we measured markers of apoptosis (B cell lymphoma 2 [Bcl-2; cleaved caspase-3) and autophagy (lysosomal-associated protein 2 [LAMP-2]). Levels of Bcl-2 (Figure 1C; $P<0.01$ ) and LAMP-2 (Figure 1E; $P<0.01$ ) were significantly decreased, while levels of cleaved caspase-3 (Figure 1D; $P<0.01$ ) were significantly increased when compared with normoxic controls. To assess whether the reduction in BAG3 levels alone was sufficient in altering the levels of markers of apoptosis and autophagy, we reduced endogenous BAG3 in NMVCs by approximately 90\% using an siRNA (Figure 1, F and G). Changes in markers of apoptosis and autophagy observed in NMVCs after H/R were recapitulated in NMVCs in which BAG3 expression was reduced by siRNA as levels of cleaved caspase-3 were increased (Figure 1I; $P<0.01$ ), while levels of Bcl-2 (Figure $1 \mathrm{H} ; P<0.01$ ) and LAMP-2 (Figure $1 \mathrm{~J} ; P<0.01$ ) were significantly reduced as compared with cells infected with adenovirus expressing GFP (Ad-GFP) control.

$B A G 3$ overexpression ameliorates changes in markers of autophagy and apoptosis. Infection of NMVCs with Ad-BAG3 3 days before evaluation modestly increased BAG3 levels (Figure 2, A and B; $P<0.01$ ) when compared with NMVCs infected with Ad-GFP. Similarly, Ad-BAG3 increased BAG3 levels in myocytes that were exposed to H/R (Figure 2, A and B; $P<0.05$ ). Ad-BAG3 had no effect on c-Jun N terminal kinase (JNK) activation or on levels of cleaved caspase-3, Bcl-2, and LAMP-2 in NMVCs incubated under normoxic conditions (Figure 2, A and C-F). By contrast, NMVCs infected with Ad-BAG3 3 days before $\mathrm{H} / \mathrm{R}$ had significantly lower levels of p-JNK $(P<0.05)$ and cleaved caspase-3 $(P<0.05)$ and increased levels of Bcl-2 $(P<0.05)$ and LAMP-2 $(P<0.01)$ when compared with control NMVCs that were infected with Ad-GFP (Figure 2 and A, C-F).

BAG3 modulates cardiomyocyte autophagy and apoptosis. To determine whether the changes in markers of autophagy represented an actual change in the amount of autophagy after H/R, NMVCs in which BAG3 levels were manipulated with Ad-BAG3 or Ad-siBAG3 were transfected with the double-labeled red fluorescent protein-GFP (RFP-GFP) microtubule-associated protein 1A/B light chain 3 (LC3-I) autophagy reporter system and then exposed to $\mathrm{H} / \mathrm{R}$. This system takes advantage of the fact that LC3-I is posttranslationally modified by a ubiquitin-like system that converts it to its lipidated LC3-II form. LC3-II is sequestered into autolysosomes in which it is degraded or recycled (15). LC3 puncta fluoresce both green and red in autophagosomes. However, in the acidic milieu of the autolysosome, the GFP fluorescence is quenched, leaving predominantly red puncta. Thus, yellow puncta represent the merged fluorescence of GFP and RFP and reflect the presence of autophagosomes, whereas red puncta represent RFP alone. In normal phagosome-lysosome fusions, there will be more red fluorescence than yellow fluorescence, whereas when autophagy is impeded with diminished phagosome-lysosome fusion, yellow fluorescence is predominant. As seen in the confocal images in Figure 3A, yellow fluorescence was more prominent in NMVCs that had undergone H/R or that had been infected with Ad-siBAG3. By contrast, RFP signals in control NMVCs and NMVCs after H/R in which BAG3 was overexpressed were more prominent, suggesting increased incorporation of LC3 into autolysosomes. The subjective evaluations of the confocal images were confirmed by counting the number of yellow and red puncta in each group (control, H/R, Ad-siBAG3, and H/R plus Ad-BAG3; Figure 3B). In addition, the average ratio of autolysosomes (red puncta) to auto- 


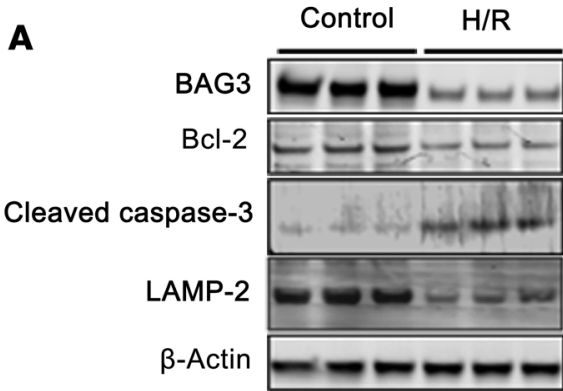

B
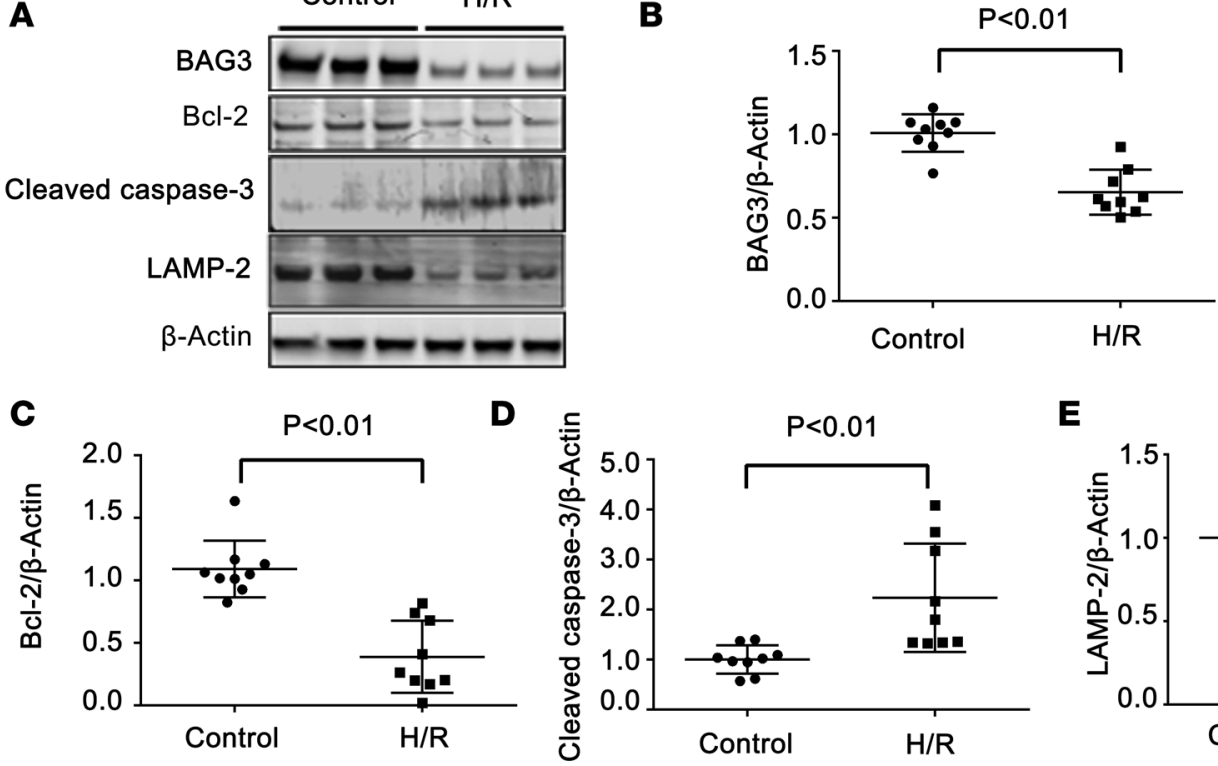

E

$\mathrm{P}<0.01$
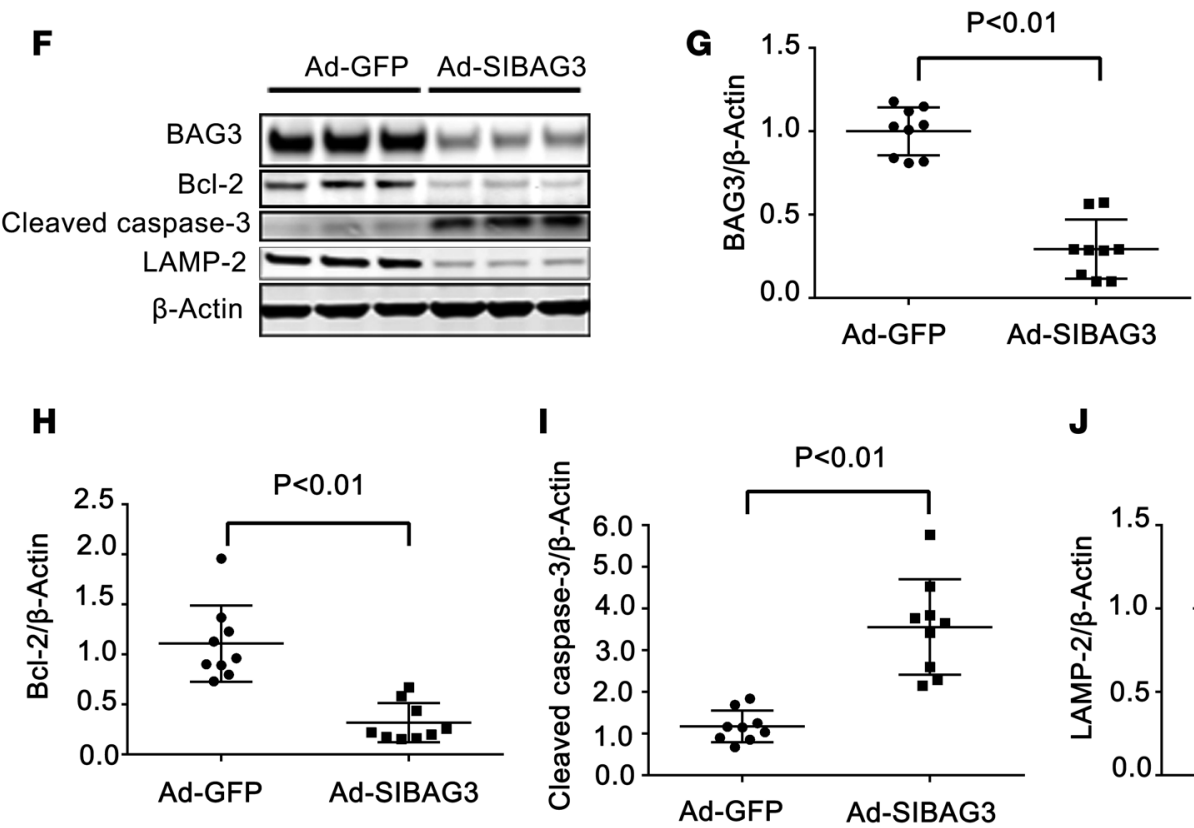

J

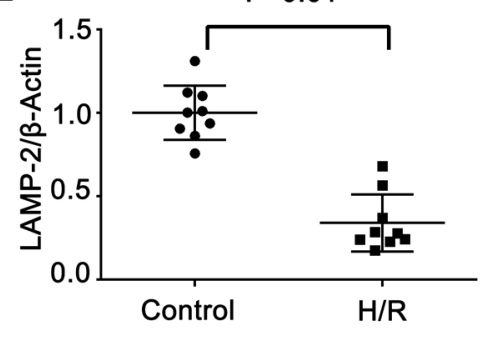


A

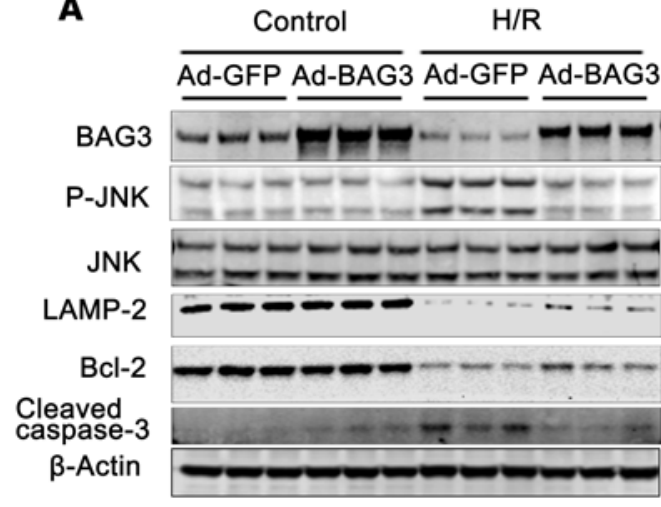

B $\stackrel{\mathrm{H} / \mathrm{R}}{\mathrm{Contro}}$

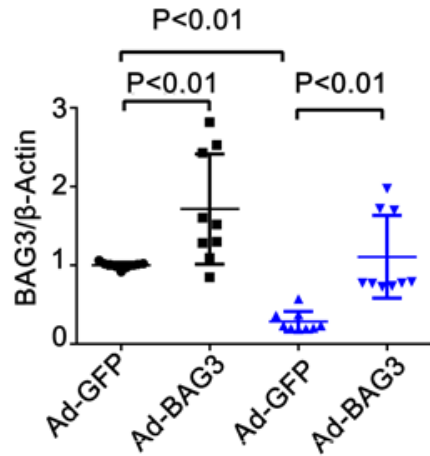

C

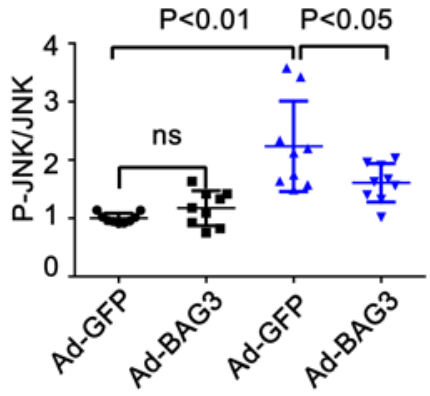

D

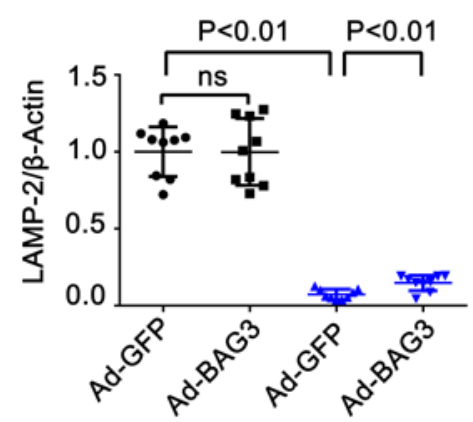

E

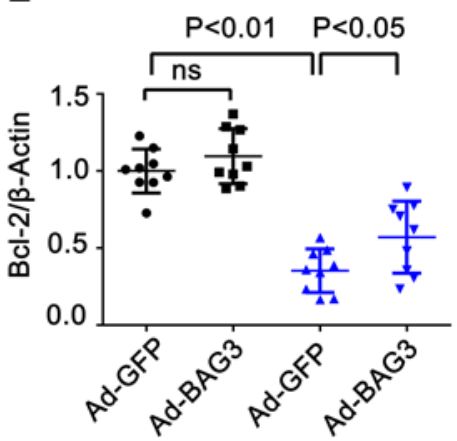

$F$

Figure 2. Overexpression of BAG3 ameliorates the changes in markers of apoptosis and autophagy in neonatal mouse ventricular cardiomyocytes after hypoxia/reoxygenation. In order to increase levels of BAC3, neonatal mouse ventricular cardiomyocytes (NMVCs) were infected with an adenovirus coupled to BAC3 (Ad-BAC3) or GFP control (Ad-GFP) and cultured for 3 days. NMVCs were then exposed to either hypoxic conditions ( $5 \%$ CO 2 and $95 \%$ nitrogen at $3 \mathrm{l} / \mathrm{min}$ ) in the absence of glucose for 14 hours at $37^{\circ} \mathrm{C}$ followed by reoxygenated for 4 hours with $5 \% \mathrm{CO}_{2}$ and $95 \%$ humidified air in medium containing glucose or normoxic conditions ( $5 \% \mathrm{CO}_{2}$ and $95 \%$ humidified air). Myocytes were then harvested, and cellular lysates were immunoblotted for BAC3, cleaved caspase-3, B cell lymphoma 2 (Bcl-2), lysosomal-associated protein 2 (LAMP-2), and the c-jun N-terminal kinase (JNK) and its phosphorylated form ( $p$-JNK). Three independent cultures of NMVCs were exposed to either hypoxia/reoxygenation or normoxia (control) in each experiment. Each experiment was then repeated 3 times in order to have $n=9$ for each intervention. (A) Representative Western blot ( $n=3$ for each group) demonstrating that BAC3 overexpression in normoxic NMVCs resulted in increased levels of BAC3 (B) but did not significantly change levels of $p-J N K(n=9)(C)$, LAMP-2 ( $n=$ 9) (D), Bcl-2 $(n=9)(E)$, or cleaved caspase-3 $(n=9)(\mathbf{F})$. By contrast, in NMVCs after hypoxia/reoxygenation, overexpression of BAC3 significantly increased BAG3 $(n=9)(\mathbf{B})$, LAMP-2 $(n=9)(\mathbf{D})$, and Bcl-2 $(n=9)(\mathbf{E})$ but decreased p-JNK $(n=9)(\mathbf{C})$ and cleaved caspase-3 $(n=9)(\mathbf{F})$. Data were normalized to the protein levels measured in NMVCs infected with control Ad-GFP and incubated under normoxic conditions. Data are presented as mean \pm SEM for continuous variables. Two-way ANOVA with Bonferroni multiple comparisons adjustments were used to assess differences across the 4 investigational groups.

phagosomes (yellow puncta) per cell was significantly reduced after $\mathrm{H} / \mathrm{R}$, a change that was mimicked by BAG3 downregulation and blunted by overexpression of BAG3. Our observations suggest that both $H / R$ and decreased levels of BAG3 decreased the amount of autophagy, whereas BAG3 overexpression restored control levels of autophagy (Figure 3C).

In order to assess the effects of BAG3 on autophagy flux using an alternative technique, cells overexpressing BAG3 or GFP were treated with bafilomycin A under conditions of normoxia or after H/R. As seen in the representative Western blots in Figure 3D and in Figure 3E, after HR there was significantly $(P$ $<0.05)$ less LC3B-II in myocytes expressing GFP when compared with myocytes overexpressing BAG3. No differences were observed when assessing LC3B-II levels in cells cultured in normoxic conditions and infected with either Ad-GFP or Ad-BAG3. These results suggest that overexpression of BAG3 during H/R increased autophagy flux when compared with cells treated with Ad-GFP control and are consistent with the tandem fluorescent results described above.

$B A G 3$ overexpression decreases apoptosis, as assessed by the number of TUNEL-positive nuclei. To confirm that the alterations in markers of apoptosis seen during manipulation of BAG3 levels by H/R, Ad-BAG3, or Ad-SiBAG3 represented a change in the actual amount of apoptosis occurring in the cells, we measured apoptosis using the TUNEL-staining assay. As seen in Figure 4, the amount of TUNEL-positive nuclei in 


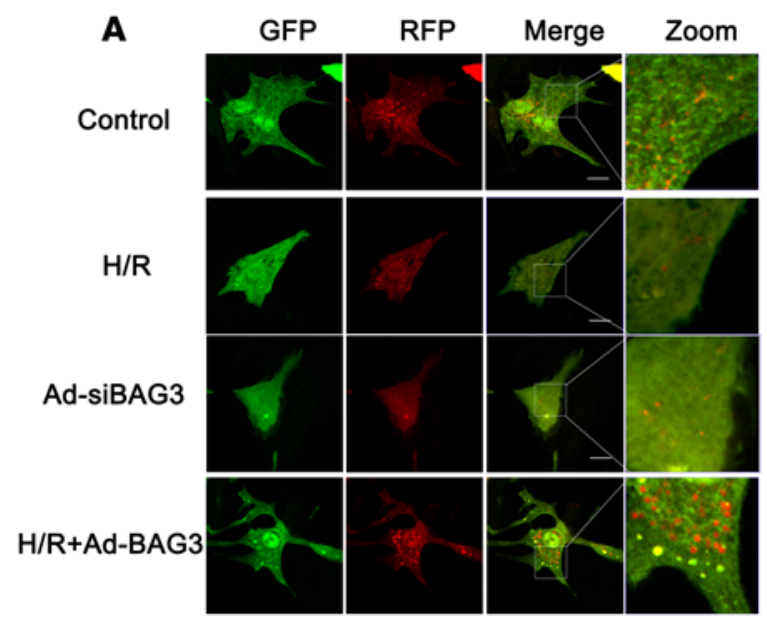

B

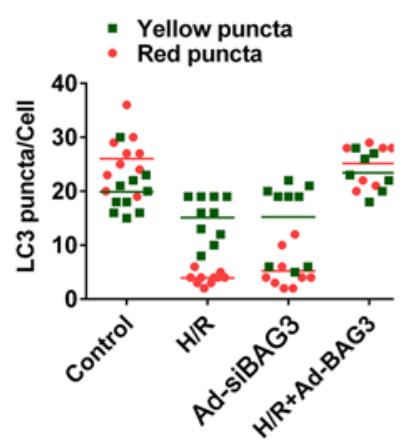

C

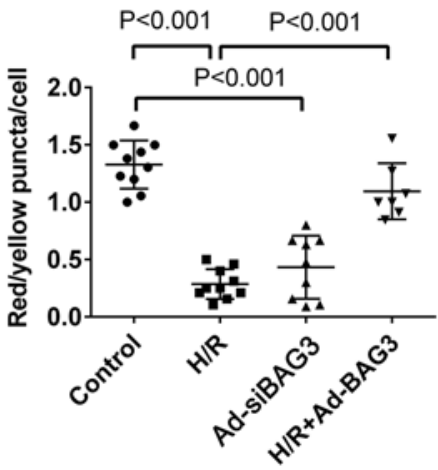

D

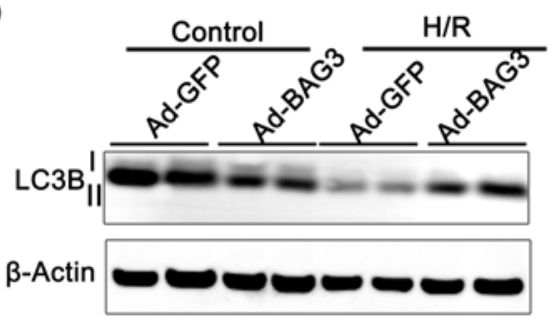

$\mathbf{E}$

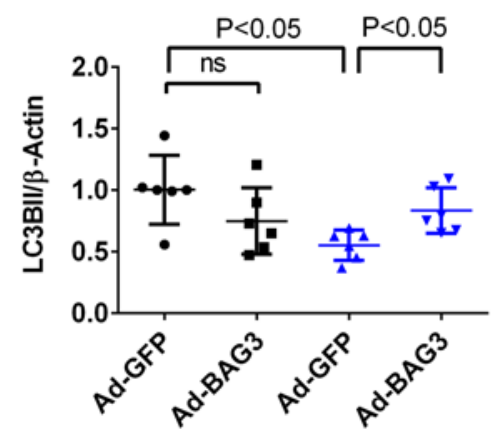

Figure 3. The levels of autophagy and apoptosis are critically dependent on BAG3 levels. To determine the effects of decreased BAC3 levels on autophagy, neonatal mouse ventricular cardiomyocytes (NMVCs) were transfected with an autophagy reporter system consisting of double-labeled Red fluorescent protein-GFP-microtubule-associated protein 1 light chain 3 (RFP-GFP-LC3-I). Both RFP (red fluorescence) and GFP (green fluorescence) can be identified in autophagosomes as yellow puncta; however, when autophagosomes fuse with lysosomes, the acidity of the autolysosome quenches the GFP fluorescence, resulting in predominantly red puncta. Independent cultures of NMVCs were cultured under normal conditions (Control, $n=9$ ), exposed to hypoxia/reoxygenation (H/R; $n=9$ ), infected with an adenovirus encapsulated with a siRNA for BAC3 (Ad-siBAC3; $n=9$ ) or exposed to $H / R$ as well as infection with an adenovirus encapsulated with BAG3 (Ad-BAG3; $n=9$ ). As shown in representative confocal images in (A), yellow fluorescence was more prominent in NMVCs in which BAG3 was reduced by either H/R or Ad- siBAG3. Despite H/R incubation, which should result in decreased BAG3 levels, RFP signals were more prominent in BAG3-overexpressed NMVCs after H/R (A), suggesting that increased incorporation of LC3 into autolysosomes is consistent with an increased level of autophagy. Scale bars: $10 \mu \mathrm{M}$. (B) The subjective evaluations of the confocal images were analyzed by counting the number of yellow and red puncta in each group (number of cells counted: $n=10$, control and H/R; $n=9$, siBAC3; $n=7, H$ /R plus Ad-BAC3). Red puncta are indicated by red dots and yellow puncta are indicated by green dots. (C) The amount of autophagy was expressed by calculating the ratio of autolysosomes (red puncta) to autophagosome (yellow puncta) per cell. Data are presented as mean \pm SEM for continuous variables. Two-way ANOVA with Bonferroni multiple comparisons adjustments was used to assess differences across the 4 investigational groups. Autophagy in NMVCs was significantly reduced after $H / R$, which was restored to normal levels by BAC3 overexpression. Reduction of BAC3 levels by Ad-siBAG3 also resulted in decreased autophagy. To provide a second assessment of autophagy flux we assessed LC-3 levels in normal control cells exposed to Ad-GFP or Ad-BAC3 and similarly treated cells exposed to H/R. (D) A representative Western blot and (E) quantification of several experiments $(n=6)$.

NMVCs under control conditions (Ad-GFP) was unaffected by increased expression of BAG3 (Ad-BAG3). However, BAG3 knockdown (Ad-siBAG3) significantly $(P<0.01)$ increased the percentage of TUNELstained nuclei. H/R effected a similar increase in the percentage of TUNEL-positive nuclei $(P<0.01)$, whereas overexpression of BAG3 (Ad-BAG3) substantially reduced $(P<0.01)$ the levels of TUNEL-positive nuclei, supporting the hypothesis that overexpression of BAG3 in cardiomyocytes could decrease the levels of apoptosis during cell stress - in this case the stress of hypoxia followed by reoxygenation.

$B A G 3$ translocates to the perinuclear and nuclear region during the stress of $H / R$. Under normal conditions, confocal imaging demonstrated that BAG3 is found predominantly in the cytoplasm of neonatal NMVCs, consistent with our previous observations (9). However, when NMVCs were exposed to H/R, BAG3 was found predominantly in the perinuclear region and in the nucleus (Figure 5A). Knocking down BAG3 by siRNA in normoxic NMVCs also resulted in the translocation of BAG3 to the perinuclear region and the nucleus (Fig- 


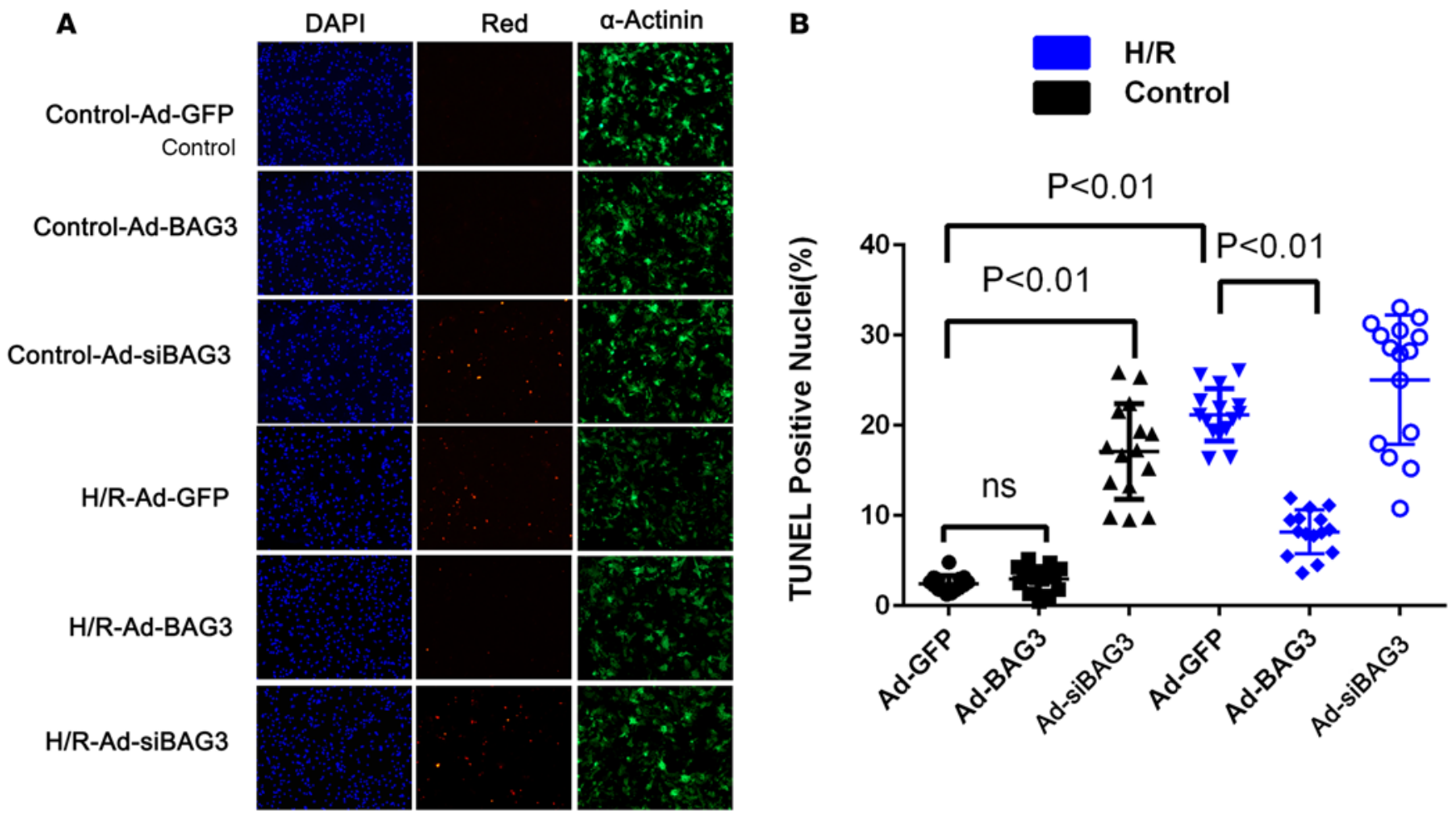

Figure 4. BAG3 knockdown and hypoxia/reoxygenation increase apoptosis in neonatal mouse ventricular cardiomyocytes, whereas overexpression of BAG3 mitigates the increase in apoptosis after hypoxia/reoxygenation. Neonatal mouse ventricular cardiomyocytes (NMVCs) were infected with an adenovirus driving the expression of BAG3 (Ad-BAG3), GFP, or a siRNA for BAG3 (Ad-siBAC3) and then cultured under hypoxic conditions (5\% $\mathrm{CO}_{2}$ and $95 \%$ nitrogen at $3 \mathrm{l} / \mathrm{min}$ ) in the absence of glucose for 14 hours at $37^{\circ} \mathrm{C}$ followed by reoxygenation for 4 hours with $5 \% \mathrm{CO}_{2}$ and $95 \%$ humidified air in medium containing glucose (hypoxia/reoxygenation [H/R]) or under normoxic (Control) condition ( $5 \% \mathrm{CO}_{2}$ and $95 \%$ humidified air). The cells were then stained with DAPI to identify nuclei; TUNEL to identify DNA fragments, a characteristic feature of apoptosis; or probed with $\alpha$-actinin to identify cardiomyocytes. Three independent cultures of NMVCs were exposed to either hypoxia/reoxygenation or normoxia (control) plus or minus infection with Ad-BAG3 or Ad-siBAG3. Each experiment was then repeated 3 times in order to have $n=9$ for each intervention. Five fields were then counted for the number of TUNEL-positive nuclei in $\alpha$-sarcomeric actinin-positive cells from each experiment using a fluorescent microscope and a $\times 20$ objective (original magnification, $\times 200)(n=$ 15). (A) Representative confocal microscopic images. (B) Data are presented as mean \pm SEM for continuous variables. Two-way ANOVA with Bonferroni multiple comparisons adjustments were used to assess differences across the 4 investigational groups. The percentage of TUNEL-positive nuclei increased significantly when BAC3 was knocked down and after H/R whereas overexpression of BAC3 attenuated the effects of H/R.

ure 5A). Cell fractionation studies confirmed the morphological findings by confocal microscopy, as BAG3 in the cytosolic fraction was decreased but BAG3 in the nuclear fraction was increased after H/R or after BAG3 was knocked down with siRNA (Figure 5, B and C).

In vivo BAG3 overexpression reduces $I / R$-induced infarct size and improves cardiac function and expression levels of autophagy and apoptosis markers. To assess whether the studies of BAG3 in NMVCs were relevant to mice in vivo, we measured ventricular function and infarct size after I/ $\mathrm{R}$ in hearts in which BAG3 was overexpressed by retro-orbital injection of rAAV9-expressing myc-tagged BAG3 under the control of a CMV promoter. As seen in Figure 6, A and B, LV ejection fraction measured 2 days after I/R in mice that had received a retro-orbital injection of rAAV9-BAG3 was significantly greater than in mice that received control rAAV9-GFP $(P<0.01)$. Consistent with the results in the neonatal myocytes after H/R (Figure 2), myocardial BAG3 levels were reduced after I/R but were enhanced after rAAV9-BAG3 (Figure 6C). The injection of rAAV9-BAG3 did not change the area at risk (AAR) (Figure 6, D and E) but significantly $(P<0.01)$ reduced infarct size at 72 hours after I/R as compared with infarct size in mice that had received an injection of rAAV9-GFP (Figure 6, D and F).

Confirming rAAV9-induced BAG3 expression in the heart, myc expression was obvious in the hearts of mice that received rAAV9-BAG3 but not in hearts of mice that had received rAAV9-GFP (Figure 7A). Consistent with the results in NMVCs after H/R (Figure 2), after I/R rAAV9-BAG3-treated hearts displayed significantly increased levels of Bcl-2 (Figure 7, A and B; $P<0.01$ ) and LAMP-2 (Figure 7, A and C; $P<0.01$ ) and decreased levels of cleaved caspase-3 (Figure 7, A and D; $P<0.01$ ) and p-JNK (Figure 7 , $\mathrm{A}$ and $\mathrm{E} ; P<0.01)$ compared with rAAV9-GFP-treated hearts after I/R. 
A

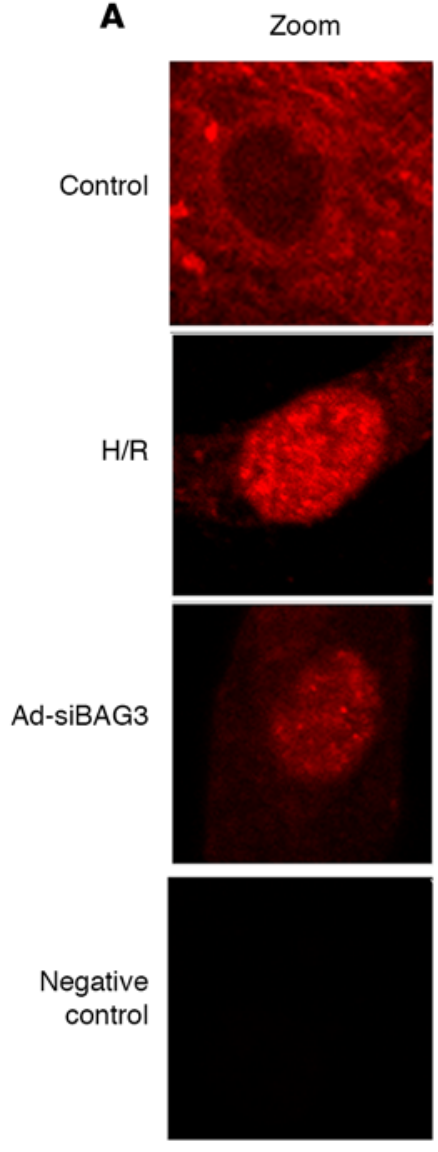

B

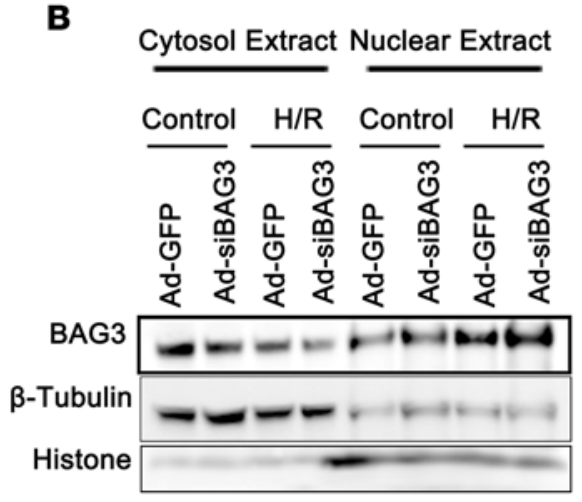

DAPI
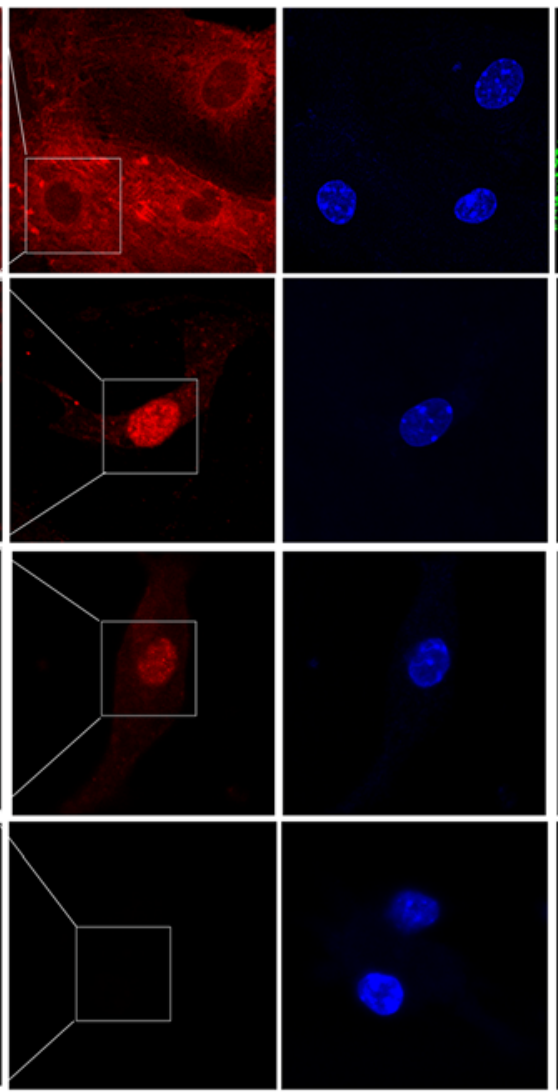

$\alpha$-Actinin
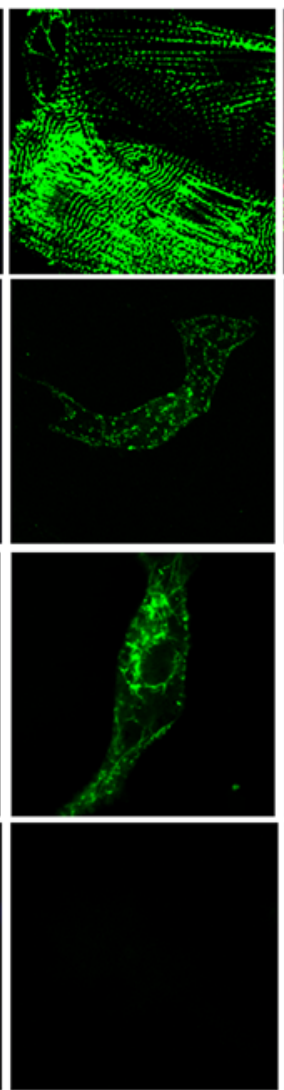

Merge
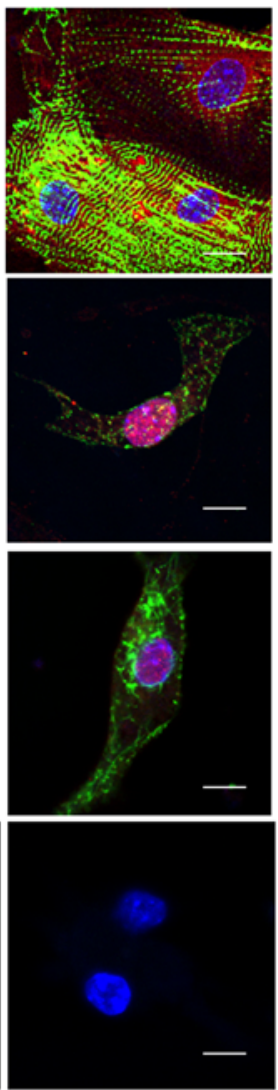

Nuclear Extract

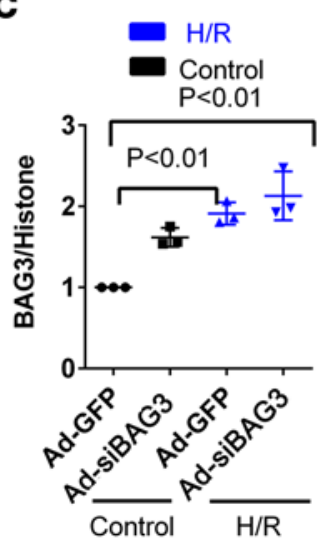

Figure 5. BAG3 translocates to the nucleus in neonatal mouse ventricular cardiomyocytes following hypoxia/reoxygenation or BAG3 knockdown. To determine whether BAG3 translocated to the nucleus during hypoxia/reoxygenation (H/R), neonatal mouse ventricular cardiomyocytes (NMVCs) were exposed to $\mathrm{H} / \mathrm{R}\left(5 \% \mathrm{CO}_{2}\right.$ and $95 \%$ nitrogen at $3 \mathrm{l} / \mathrm{min}$ in the absence of glucose for 14 hours at $37^{\circ} \mathrm{C}$ followed by reoxygenated for 4 hours with $5 \% \mathrm{CO}_{2}$ and $95 \%$ humidified air in medium containing glucose) or normoxic conditions and infected with an adenovirus encapsulated with a siRNA (Ad-siBAC3) to decreased BAC3 levels or with an adenovirus encapsulated with GFP (Ad-GFP control). Three independent cultures of NMVCs were used in each experiment in order to have $n=3$ for each intervention. NMVCs were fixed and stained with a BAC3 antibody, an $\alpha$-actinin antibody, or the nuclear counter-stain DAPI. (A) Representative confocal images of BAG3 localization in NMVCs that were positive for DAPI and $\alpha$-actinin. Scale bars: $10 \mu M$. Both H/R and BAG3 knockdown with Ad-siBAG3 appear to increase the presence of BAG3 in the nucleus when compared with NMVCs treated under control conditions. (B) To confirm the visual observation that BAG3 translocated to the nucleus during the stress of H/R or after knockdown with siBAG3, cytoplasm and nuclear fractions of NMVCs were prepared using a NE-PER nuclear and cytoplasmic extraction kit (ThermoFisher Scientific). Western blot of BAG3 in cytoplasmic and nuclear fractions obtained from Ad-GFP and BAC3 knockdown (Ad-siBAG3) NMVCs subjected to normoxic (control) or H/R incubations demonstrates that $\beta$-tubulin was present in all 3 cytosol fractions but not in the nuclear fraction, whereas histone was present in all 3 nuclear fractions but not in the cytosol, suggesting adequate separation of the two fractions. (C) Quantification of Western blots of nuclear extracts demonstrates that BAC3 levels (normalized to histone) in nuclear extracts increased following BAG3 knockdown (Ad-siBAG3) or after H/R. Two-way ANOVA with Bonferroni multiple comparison adjustments were used to assess differences across the 4 investigational groups. 
A

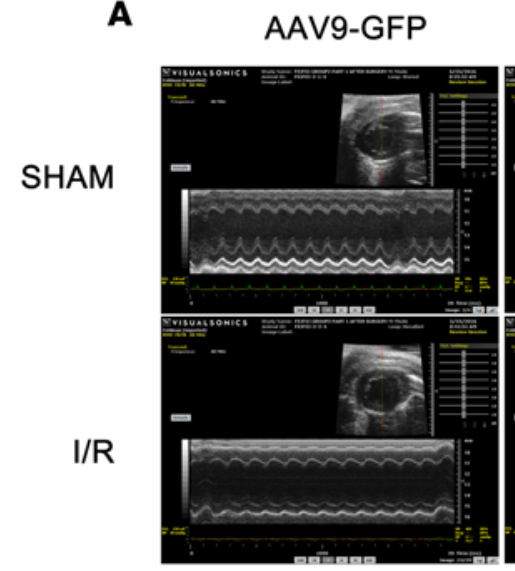

AAV9-BAG3

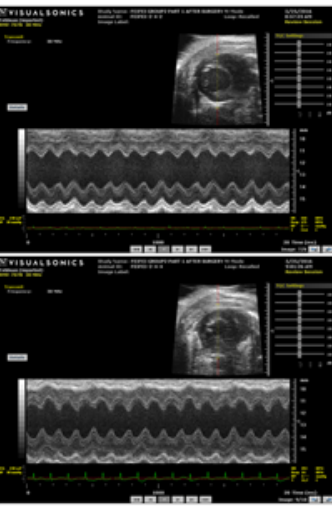

I/R

C

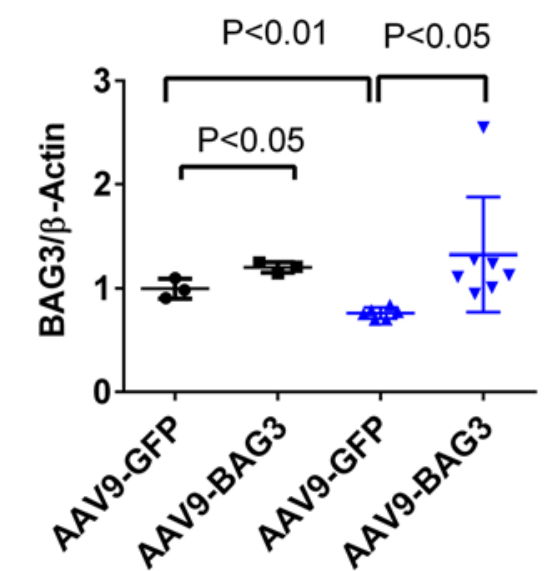

$\mathbf{E}$

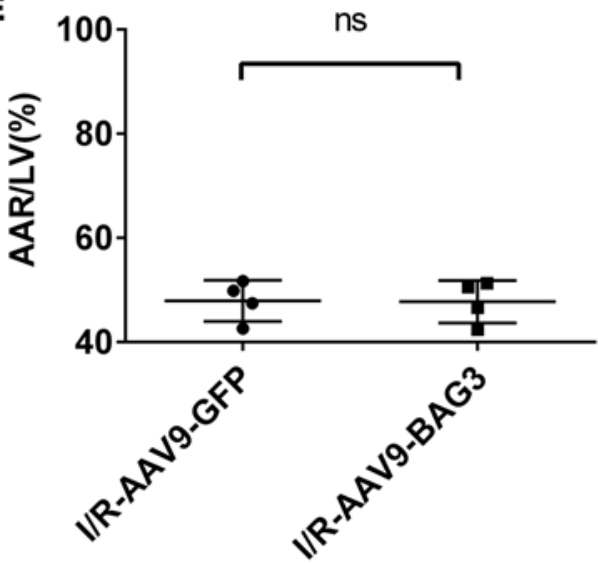

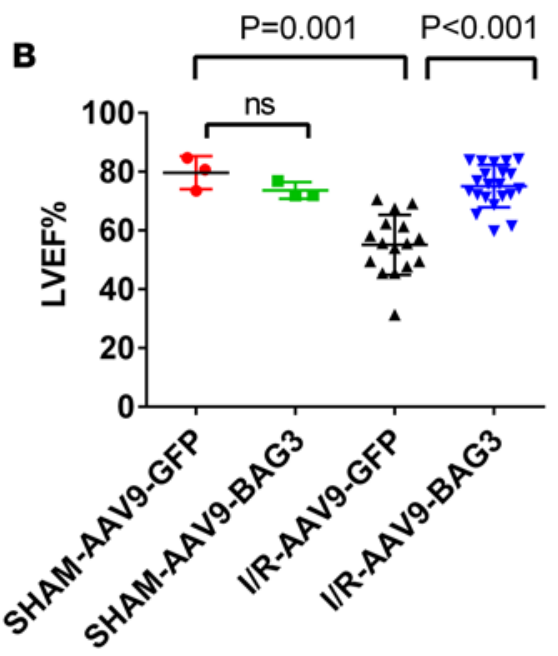

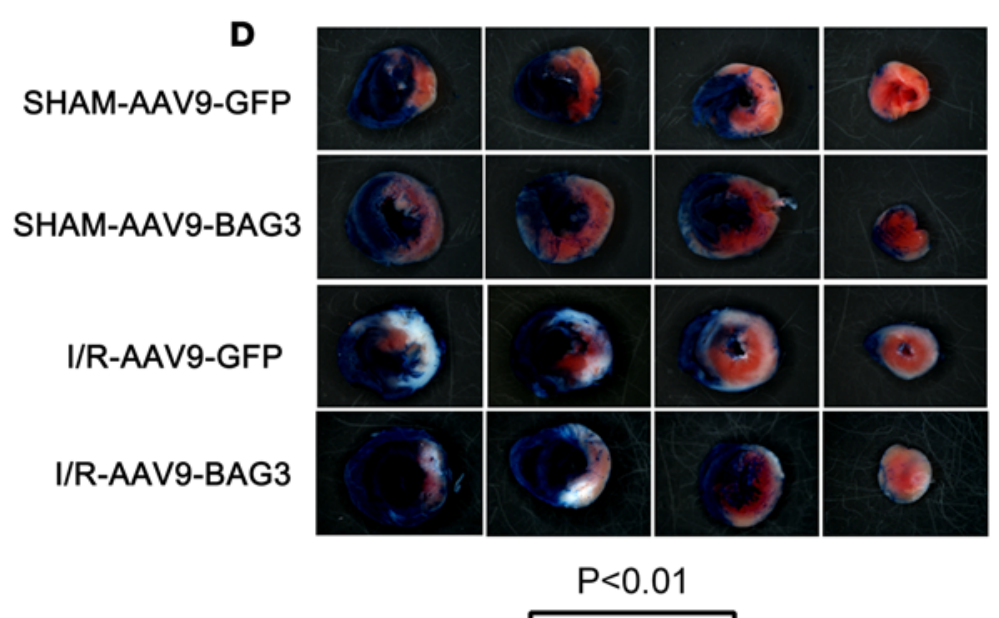

$\mathbf{F}$

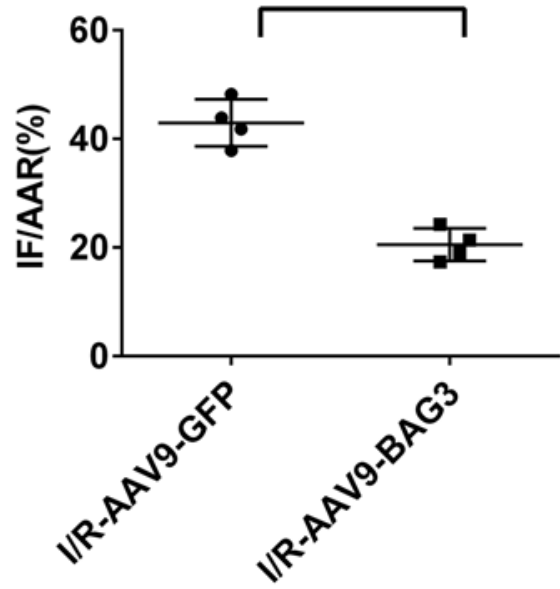

Figure 6. BAG3 preserves function and limits infarct size after ischemia/reperfusion. Male FVB mice (8-10 weeks old) underwent retro-orbital injection with an adeno-associated virus serotype 9 (rAAV9) expressing either GFP or BAC3. Three weeks later, mice were randomly assigned to coronary ligation for 30 minutes followed by reperfusion or sham surgery. Seventy-two hours later, mice underwent echocardiography and sacrifice. Forty-three mice were randomly assigned to Sham-GFP $(n=3)$, Sham-rAAV9-BAG3 $(n=3)$, I/R-rAAV9-GFP $(n=16)$, or I/R-rAAV9-BAG3 ( $n=21)$ in 3 different studies and underwent initial echocardiography. Echocardiographic data represented aggregate information from Western blot analysis of BAC3 in mice injected with rAAV9-BAG3 or rAAV9-GFP prior to ischemia/reperfusion (I/R) or sham $(C, n=19)$; determination of infarct size by triphenyltetrazolium staining in mice injected with either rAAV9-GFP or rAAV9-BAG3 prior to I/R $(\mathbf{D}, n=10)$; and Western blot analysis of markers of autophagy and apoptosis in mice injected with rAAV9-GFP or rAAV9-BAG3 prior to I/R (Figure $7 ; n=9$ ). The difference between the number of animals undergoing echocardiography and the analysis reported in the figures is due to loss of animals prior to subsequent analysis, as detailed in Methods. (A) Representative M-mode echocardiograms of hearts expressing either GFP or BAC3 after I/R or sham. (B) Left ventricular ejection fraction (LVEF) of sham-operated or I/R hearts expressing either GFP or BAG3; Sham-GFP $(n=3)$, Sham-BAC3 $(n=3)$, I/R-GFP $(n=16)$, I/R-BAC3 $(n=21)$. (C) BAC3 levels from heart homogenates from sham-operated and I/R hearts expressing GFP or BAG3; Sham-GFP $(n=3)$, Sham-BAG3 $(n=3)$, I/R-GFP $(n=6)$, I/R-BAG3 $(n=7)(D)$ In a separate experiment, 72 hours after 
I/R, Evans Blue dye was injected into hearts, and they were excised and stained with triphenyltetrazolium. The Evans Blue-stained area (area not at risk), triphenyltetrazolium-negative area (infarcted myocardium [IF]), and area at risk ([AAR], triphenyltetrazolium-negative and -positive areas) were measured. AAR was expressed as percentage of total LV (\%AAR/LV). Infarcted myocardium (triphenyltetrazolium-negative area) was expressed as percentage of AAR (\%IF/AAR). (E) There were no differences in AAR (AAR/LV\%) between after I/R hearts expressing GFP $(n=4)$ or BAC3 $(n=4)$, (F) although infarct size (IF/AAR\%) was significantly smaller in after I/R hearts infected with rAAV9-BAC3 $(n=4)$ when compared with hearts infected with rAAV9-GFP $(n=$ 4). Two-way ANOVA with Bonferroni multiple comparisons adjustments assessed differences across the 4 investigational groups. A Student's 2-tailed $t$ test was used to analyze data comparing two groups.

\section{Discussion}

BAG3 is an evolutionarily conserved protein that was first recognized for its antiapoptotic activity, although it also serves as a cochaperone of both the constitutively and nonconstitutively expressed chaperone protein heat shock protein 70 (Hsc/Hsp70) in facilitating selective macroautophagy (1). BAG3 has come to the attention of investigators focused on the heart due to the observation that mutations in BAG3 lead to familial dilated cardiomyopathy (2), the finding that BAG3 modulates excitation-contraction coupling in the heart (9), and our recent observation that BAG3 promoted mitochondrial degradation through the autophagy-lysosome pathway and through direct interactions with mitochondria (12). Because disruption of the normal removal of damaged and dysfunctional mitochondria plays a pivotal role in reperfusion injury following ischemia, we hypothesized that alterations in the expression or function of BAG3 might play a role in reperfusion injury. To test this hypothesis, we first evaluated the role of BAG3 in cultured NMVCs exposed to the stress of $\mathrm{H} / \mathrm{R}$, a surrogate for I/R, and then sought to confirm our observations in adult mice with LV dysfunction after I/R.

We demonstrate that (a) levels of BAG3 were substantially reduced after both H/R in NMVCs and $\mathrm{I} / \mathrm{R}$ in the infarct border zone of the ventricular myocardium of mice; (b) the reduced levels of BAG3 in NMVCs after $\mathrm{H} / \mathrm{R}$ and in mouse heart muscle after I/R were associated with changes in the levels of markers of autophagy, apoptosis, and lysosomal function, including increased levels of cleaved caspase-3 and decreased levels of Bcl-2 and LAMP-2; (c) BAG3 knockdown with an siRNA (siBAG3) in NMVCs resulted in an apoptosis/autophagy biomarker phenotype that exactly mirrored that seen in NMVCs after H/R; (d) overexpression of BAG3 by an adenovirus (Ad-BAG3) in NMVCs normalized the alterations of biomarkers for apoptosis and autophagy after H/R; and (e) a rAAV9-expressing BAG3 under the control of a CMV promoter (rAAV9-BAG3) significantly enhanced LV function and decreased infarct size after I/R in mice, while also modifying the levels of biomarkers for autophagy and apoptosis commensurate with those seen in NMVCs. In aggregate, these results support the hypothesis that normal levels of BAG3 are necessary for maintaining cardiac homeostasis during the stress of hypoxia/ischemia and reperfusion.

We chose a group of biomarkers for autophagy, apoptosis, and lysosomal function that had previously been shown to reflect biological processes that were pivotal in the pathobiology of both apoptosis and autophagy and that had been used previously for identifying changes in these processes during stress. For example, LAMP-2 is a lysosomal marker that is an important determinant of autophagosome-lysosome fusion (16-19); Bcl-2 stimulates autophagy by disrupting its association with Beclin 1, leading to the activation of the Beclin 1-associated class III ptdlns3K complex (20), while also playing a role in limiting apoptosis when bound to the Bcl-2-binding site of BAG3; and cleaved caspase-3 is a protease responsible for chromatin margination, DNA fragmentation, and nuclear collapse during the execution phase of apoptosis (21, 22). However, considerable controversy has surrounded the use of biomarkers for measuring autophagy, because it is a dynamic multistep process that begins with the formation of a phagophore and proceeds through the maturation of the phagophore as it recruits membranes from different intracellular sources and accumulates targeted proteins, and, finally, it fuses with lysosomes to form an autolysosome in order to begin the process of protein digestion (1). Therefore, to better assess the effects of both diminished and enhanced levels of BAG3 on autophagy, we used an autophagy reporter system consisting of double-labeled microtubule-associated protein LC3-I, as recommended by the recent consensus guidelines for the use and interpretation of assays for monitoring autophagy (23).

The tandem fluorescent system takes advantage of the fact that LC3-I is posttranslationally modified by a ubiquitin-like system that converts it to its lipidated LC3-II form, which is anchored to the outer and inner membranes of autophagosomes. LC3-II is sequestered into autolysosomes where it is degraded or recycled (15). LC3 puncta fluoresce both green and red in autophagosomes. However, in the acidic milieu of the autolysosome, the GFP fluorescence is quenched leaving predominantly red puncta. Our studies demonstrated that both H/R and BAG3 knockdown resulted in decreased levels of autophagy, whereas BAG3 overexpres- 
A

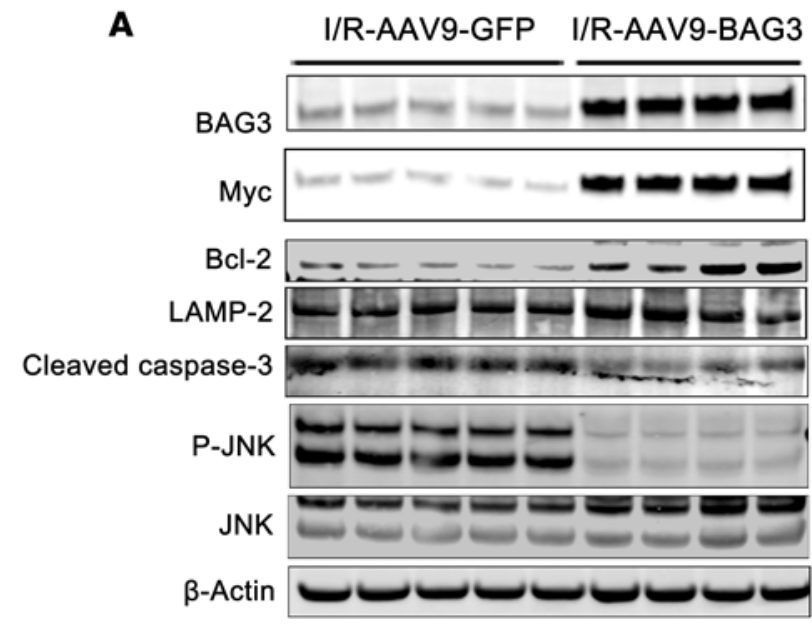

B

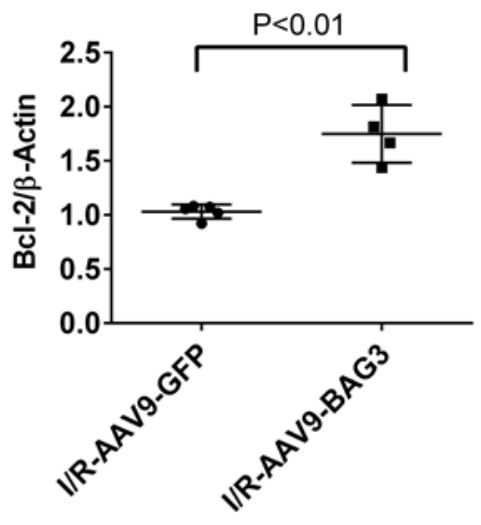

C

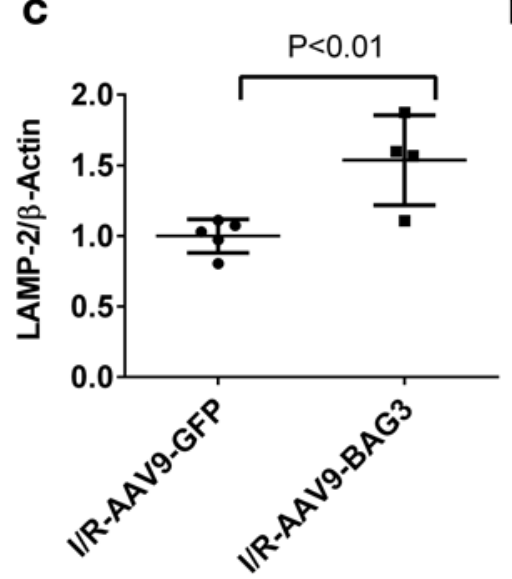

D

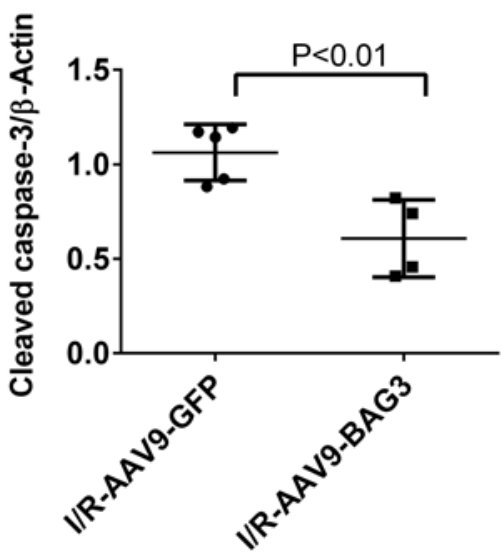

$\mathbf{E}$

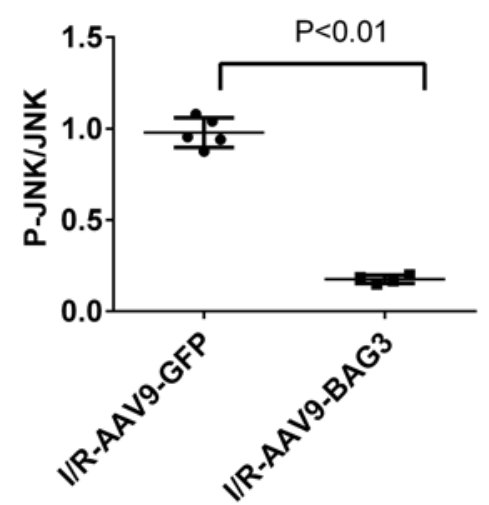

Figure 7. Overexpression of BAG3 in hearts ameliorates deleterious changes in markers of apoptosis and autophagy after ischemia/reperfusion. Heart tissue was obtained from the border zone of mice that had been injected in the retro-orbital plexus with either rAAV9-GFP $(n=5)$ or rAAV9-BAC3 $(n=4)$ 3 weeks prior to ischemia/reperfusion (I/R) (ligation of the left coronary artery for 30 minutes followed by 72 hours of reperfusion) or sham surgery. (A) Western blot probed for markers of apoptosis and autophagy using specific antibodies for cleaved caspase-3, B cell lymphoma 2 (Bcl-2), lysosomal-associated protein 2 (LAMP-2), and the phosphorylated form of $\mathrm{c}-\mathrm{Jun} \mathrm{N}$ terminal kinase ( $\mathrm{pJNK}$ ). Myc levels were determined to document expression of rAAV9BAC3, and $\beta$-actin served as a loading control. Beneficial changes in biomarkers of autophagy and apoptosis in adult hearts with BAC3 overexpression after I/R were consistent with those observed in BAC3-overexpressed NMVCs subjected to hypoxia/reoxygenation (Figure 2). Quantification of Western blots for Bcl-2 (B), LAMP-2 (C), cleaved caspase-3 (D), and p-JNK (E). Data were normalized to the values measured for after I/R hearts expressing GFP. A Student's 2-tailed $t$ test was used to analyze data when comparing two groups.

sion restored the level of the autophagy process. These results are consistent with an earlier report by Ma et al., demonstrating that I/R injury impairs autophagosome clearance mediated in part by ROS-induced decline in LAMP-2 (24). Similarly, we used TUNEL staining to confirm that the changes identified in levels of cleaved caspase 3 and Bcl-2 were representative of a change in the amount of apoptosis.

The role of autophagy $(25,26)$ and apoptosis $(27,28)$ in the development of cardiovascular disease and in particular in the development of heart failure has been well recognized and is the subject of recent reviews. In fact, modest overexpression of active caspase leads to the development of heart failure (28). However, a pivotal role for BAG3 in regulating cardiac protection and its associated effects on autophagy and apoptosis have not been previously recognized. Importantly, while restitution of diminished levels of BAG3 after H/R or I/R lead to salutary effects in cells or tissues that have been stressed, BAG3 appears to have no untoward effects on either autophagy or apoptosis when BAG3 levels are increased in cells or hearts that have not been exposed to stress, suggesting that attempts to increase BAG3 levels could provide a unique and important therapeutic approach to cardiac protection.

An interesting finding was that JNK was activated (p-JNK) when BAG3 levels decreased during H/R or I/R, but the level of activation decreased when BAG3 levels were increased by Ad-BAG3 or rAAV9-BAG3 
in NMVCs or the adult heart, respectively. JNK belongs to the MAPK family of kinases but is differentiated from other kinases in that it belongs to the group of MAPKs (p-JNK, ERK1/2, and p38) that can phosphorylate non-kinase substrates, including transcription factors and scaffolding proteins (29). Previous studies have demonstrated that JNK is activated in the heart during reperfusion following ischemia but not by ischemia alone (30). Furthermore, studies in nonmyocytes suggest that activation of JNK enhanced BAG3 gene expression, whereas JNK inhibitors decreased BAG3 expression $(31,32)$. Therefore, there may be a feedback loop that decreases JNK activation in the heart when BAG3 levels are high and increases JNK activation when BAG3 levels are low. However, the interaction between BAG3 and JNK is highly complex (33), and further studies are required to clarify the relationship between BAG3 and JNK in the heart.

Another interesting observation was that BAG3 translocated from the cytoplasm to the nucleus during the stress of hypoxia and reoxygenation. While BAG3 translocation has not been reported in myocytes, the finding is consistent with a previous study demonstrating that BAG3 can be found in the nucleus of human glial cells, resulting in its ability to stimulate its own transcription through a positive feedback loop involving its 5'-untranslated sequence (34). Thus, in addition to the increasing list of functions for BAG3 in the heart, it appears that BAG3 can also regulate gene expression. This plasticity is due in part to the presence of numerous protein-binding motifs within the BAG3 protein.

Despite successful efforts to limit the time between the onset of coronary obstruction and coronary intervention in patients with an acute myocardial infarction, myocardial damage due to reperfusion injury remains a major clinical problem that has failed to be influenced by multiple pharmacologic approaches. The findings that BAG3 levels are reduced during the stress of $\mathrm{H} / \mathrm{R}$ in vitro or $\mathrm{I} / \mathrm{R}$ in vivo and that overexpression of BAG3 reduces infarct size and improves LV function after I/R in mice suggest that BAG3 could provide a therapeutic target for cardiac protection. We recognize that biological differences exist between mice and humans (35), and it will be important to demonstrate that similar salutary benefits of enhancing BAG3 levels can be seen in a large animal model of I/R injury. Nonetheless, our results suggest that moving from evaluations in mice to studies in large animals with ischemia and reperfusion, the next step in translational science paradigm, would be warranted.

\section{Methods}

Animal protocols. Neonatal mice were obtained from female FVB mice within 3 days of birth (Jackson Laboratory). Eight- to ten-week-old male FVB mice (Jackson Laboratory) were used for assessment of infarct size after 30 minutes of coronary ligation and subsequent reperfusion as described previously (36). Sham-operated control animals were treated in an identical manner except, that the LAD was not ligated.

A total of 43 mice were randomly assigned to Sham-rAAV9-GFP $(n=3)$, Sham-rAAV9-BAG3 $(n=3)$, I/R-rAAV9-GFP $(n=16)$, or I/R-rAAV9-BAG3 $(n=21)$ groups in the context of 3 different studies and underwent echocardiography as an initial evaluation in each. Thus the echocardiographic data represented aggregate information from mice from (a) Western blot analysis of BAG3 levels in mice injected with rAAV9-BAG3 or rAAV9-GFP prior to I/R or sham surgery, as shown in Figure 6C $(n=19)$; (b) determination of infarct size by triphenyltetrazolium (TTC) staining in mice injected with either rAAV9-GFP or rAAV9-BAG3 prior to I/R, as shown in Figure 6D $(n=8)$; and (c) Western blot analysis of markers of autophagy and apoptosis in mice injected with rAAV9-GFP or rAAV9-BAG3 prior to I/R, as shown in Figure $7(n=9)$. A statistically significant difference between groups was evident if the echocardiographic data from each experiment were independently assessed. The difference between the total number of animals undergoing echocardiography after I/ $\mathrm{R}$ and the analysis reported in the figures is due to the death of one rAAV9-GFP-treated mouse after echocardiography from the group reported in Figure 6C, the exclusion of one animal from further analysis in the rAAV9-BAG3 group in Figure 6C, and the exclusion of one animal from further analysis in the rAAV9-BAG3 group in Figure 7 due to an absence of myc (by Western blot), which was indicative of a failure of uptake of the rAAV9-BAG3 from the retro-orbital plexus. The decision to exclude these animals was made prior to the start of the studies and was based on preliminary data obtained in our laboratory demonstrating a modest failure rate of the rAAV9 vector due to either technical difficulties or the presence of antibodies to AAV. In addition, 4 animals in the group assigned to assessment of infarct size did not provide data points due to a failure to adequately cannulate the coronary artery, resulting in a totally blue heart. This technical failure occurred equally in both treatment groups and was seen exclusively in the early learning curve of the study. 
Preparation of primary NMVCs. NMVCs were isolated from 1- to 3-day-old FVB mice using a Pierce Primary Cardiomyocyte Isolation Kit (catalog no. 88281, ThermoFisher Scientific) according to the manufacturer's instructions. Myocytes were seeded into each well of 6-well plates at a concentration of $2 \times 10^{6}$ cells per plate and cultured in DMEM (GIBCO, ThermoFisher Scientific) with $10 \%$ fetal bovine serum (Denville Scientific Inc.) and 1\% penicillin-streptomycin (ThermoFisher Scientific). After 24 hours, the complete medium was replaced with fresh DMEM containing cardiomyocyte growth supplement at $37^{\circ} \mathrm{C}$ and $5 \% \mathrm{CO}_{2}$.

Construction and use of BAG3 adenovirus. Ad-GFP, Ad-BAG3, or Ad-siBAG3 was constructed using the BD Adeno-X Expression System, 2PT3674-1, and BD Knockout RNAi Systems, PT3739 (BD Biosciences-Clontech) as previously described (9). Forty-eight hours after isolation, NMVCs were infected with adenovirus at a multiplicity of infection of 8 . NMVCs were exposed to adenovirus overnight, after which media were aspirated and fresh media were applied. Media were changed daily. Experiments were then performed 72 hours after infection.

$H / R$. NMVCs were subjected to $\mathrm{H} / \mathrm{R}$ as described previously (37) with modifications. In brief, NMVCs were exposed to humidified $5 \% \mathrm{CO}_{2} / 95 \% \mathrm{~N}_{2}$ for 14 hours at $37^{\circ} \mathrm{C}$ and incubated in glucose-free medium. Cells were then reoxygenated with $5 \% \mathrm{CO}_{2} / 95 \%$ humidified air for 4 hours in medium containing glucose. Medium was replaced daily. In a separate group of experiments, Bafilomycin $\mathrm{A}_{1}$ (Selleckchem) was added to the incubation medium (final concentration of $50 \mathrm{nM}$ ) at the time of reoxygenation to neutralize lysosome $\mathrm{pH}$ as well as to block lysosome-autophagosome fusion.

Immunoblotting. Hearts were excised, and left ventricles were separated into infarct border ( $3 \mathrm{~mm}$ of proximal most end of apex) and remote zones (proximal septum). Tissues were quickly frozen in liquid nitrogen and stored at $-80^{\circ} \mathrm{C}$ until use. Membrane proteins were prepared as described previously (38). In brief, tissue was lysed in buffer (Cell Signaling Technologies) containing protease and phosphatase inhibitor cocktail (ThermoScientific) and homogenized with beads in a Bullet Blender (Next Advance). NMVCs were rinsed with ice-cold PBS, collected, and lysed in buffer (38). After centrifugation at $13,000 \mathrm{~g}$ for 5 minutes at $4^{\circ} \mathrm{C}$, the supernatant was collected and protein levels were determined by Bradford assay (Bio-Rad). Equal amounts of protein $(90 \mu \mathrm{l})$ were mixed with $30 \mu \mathrm{l}$ of $4 \times$ NuPAGE SDS sample buffer (ThermoFisher) and $15 \mu 1$ of $10 \times$ NuPAGE reducing agent (ThermoFisher), boiled, separated on NuPAGE Novex 4\%-12\% Bis-Tris Protein Gels (ThermoFisher) using the NuPAGE electrophoresis system (ThermoFisher), and transferred to nitrocellulose membranes (LiCor). Membranes were blocked in Odyssey blocking buffer (LiCor) for 1 hour at room temperature before incubated with primary antibodies overnight. The membranes were washed with $1 \times$ PBS-T $(0.1 \%$ Tween 20$)$ and incubated with secondary antibody for 1 hour at room temperature. Protein band signals were detected with an Odyssey scanner. Primary antibodies used were Myc (catalog no. 2276; Cell Signaling Technologies), BAG3 (catalog no. 10599-1-AP, Protein Tech), Bcl-2 (catalog no. 3498; Cell Signaling Technologies), LAMP-2 (catalog no. PA1-655; ThermoFisher), cleaved caspase-3 (catalog no. 9661; Cell Signaling Technologies), JNK (catalog no. SC-7345; Santa Cruz Biotechnology), p-JNK (catalog no. 4671; Cell Signaling Technologies), histone, (catalog no. 4499; Cell Signaling Technologies), $\beta$-tubulin, (catalog no. 2128; Cell Signaling Technologies), $\beta$-actin (catalog no. SC-47778; Santa Cruz Biotechnology), and LC3-B (catalog no. NB00-2220; Novus Biologicals). Secondary antibodies used were goat anti-mouse IRDye 800 (catalog no. 926-32210; LiCor Biosciences) and IRDye 680 goat anti-rabbit (catalog no. 926-68071; Licor Biosciences).

Confocal microscopy. Confocal microscopy was used to detect BAG3 localization in adult cardiomyocytes as described previously (9). Briefly, NMVCs were isolated and plated on laminin-coated 4-well chamber slides (Lab-Tek). BAG3 was identified using a primary rabbit antibody (1:200; catalog no. 10599-1-AP; Proteintech) $(39,40)$, and $\alpha$-sarcomeric actinin was identified using a mouse antibody (1:200, catalog no. A7732; Sigma-Aldrich). The secondary antibody used was Alexa Fluor 594-labeled goat anti-rabbit antibody (1:500; catalog no. A11012; Invitrogen), and mounting media contained DAPI (catalog no. H-1200; Vector Laboratories). A Carl Zeiss 710 confocal microscope $(\times 63$ oil objective) with ZEN software was used for imaging for BAG3 (594 nm ex., $667 \mathrm{~nm} \mathrm{em}$.), $\alpha$-actinin (catalog no. A11001, Invitrogen; $488 \mathrm{~nm}$ ex., $543 \mathrm{~nm}$ em.), and DAPI (405 nm ex., $495 \mathrm{~nm}$ em.). Total laser intensity and photomultiplier gain were set constant for all groups and settings, and data were verified by two independent observers who were blinded to the experimental group. A minimum of 3 coverslips were used for each experimental group, and at least 3 cell images were acquired from each coverslip.

Autophagy RFP-GFP-LC3 reporter system. Isolated NMVCs were infected with an adenovirus expressing mRFP-GFP-LC3 (provided by M. Madesh) at multiplicity of infection of 1, as described previously (41). NMVCs were subjected to H/R 48 hours after infection and then fixed with paraformaldehyde in PBS (9). 
After rinsing with PBS, the cells were permeabilized with $0.3 \%$ Triton X-100 in 10\% normal goat serum blocking solution (Invitrogen, Life technologies) for 60 minutes. Coverslips were mounted to slides with Hardset anti-fade mounting medium (Vector Laboratories), and confocal imaging was performed as described above (mRFP $594 \mathrm{~nm}$ ex., $667 \mathrm{~nm}$ em.; GFP $488 \mathrm{~nm}$ ex., $543 \mathrm{~nm}$ em.). The puncta of 7-10 cells in each experimental group were counted after obtaining digital images. The number of yellow puncta in the merged channel represented the number of autophagosomes. The number of autolysosomes (as a result of autophagosome-lysosome fusion) were represented by the number of red puncta as described previously $(42,43)$.

TUNEL assay. Apoptosis was quantified in NMVCs using the TUNEL kit (Roche) according to the manufacturer's instructions. In brief, immediately after H/R treatment, NMVCs were rinsed with PBS and fixed with $4 \%$ paraformaldehyde. The cells were then permeabilized with $0.3 \%$ Triton X-100 in 10\% normal goat serum for 2 minutes on ice followed by incubation in TUNEL reaction mixture for 60 minutes at $37^{\circ} \mathrm{C}$ in the dark. After the cells were blocked with 10\% normal goat serum (Invitrogen, Life Technologies) for 1 hour at room temperature, they were incubated with Alex Fluor 488-labeled goat anti-mouse antibody (1:500, catalog no. A11001; Invitrogen) at room temperature in the dark for 1 hour. The cells were then mounted with hardset anti-fad mounting medium, including DAPI (Vector Laboratories) and covered with a glass cover. The TUNEL-positive nuclei and the total number of nuclei were counted in $\alpha$-sarcomeric actinin-positive cells in at least 5 fields in 3 independent experiments. More than 2,250 nuclei were counted under both $\mathrm{H} / \mathrm{R}$ and normal conditions. A Nikon Eclipse 80 fluorescence microscope was used for visualizing the TUNEL-positive nuclei, and NIS Elements software was used to capture the images.

Cell fractionation. The cytoplasm and nuclear fractions of NMVCs were prepared using a NE-PER nuclear and cytoplasmic extraction reagent kit (ThermoFisher Scientific) according to the manufacturer's instructions. The specificity of the fractions was confirmed by the presence of $\beta$-tubulin predominantly in the cytosolic extract and histone predominantly in the nuclear fraction. Both the cytoplasmic and nuclear extractions were stored at $-80^{\circ} \mathrm{C}$ until use for Western blot.

Construction and administration of rAAV9-BAG3. A sequence encoding the murine myc-tagged BAG3 (NCBI accession BC145765) was inserted into a pAAV vector that contained a CMV promoter (Vector Biolabs). The construct was then packaged into AAV-9 by transfection of HEK293 cells, and viral particles were purified by $\mathrm{CsCl}_{2}$ centrifugation (Vector Biolabs). Recombinant AAV9-BAG3 also expressed GFP; however, GFP was not in sequence with BAG3. Fidelity of the clone and the final vector were confirmed by sequencing. Both $\mathrm{I} / \mathrm{R}$ mice and sham mice were randomly assigned to receive either 60-80 $\mu 1 \mathrm{rAAV} 9$ BAG3 $\left(5.0 \times 10^{13}\right.$ to $6.5 \times 10^{13}$ genome copies $\left.[\mathrm{GC}] / \mathrm{ml}\right)$ or rAAV9-GFP control $\left(3.1 \times 10^{12} \mathrm{GC} / \mathrm{ml}\right)$ in sterile PBS at $37^{\circ} \mathrm{C}$ by injection into the retro-orbital venous plexus as described previously (44).

Echocardiography. Global LV function was evaluated in all mice after light sedation ( $2 \%$ isoflurane) using a VisualSonics Vevo 770 imaging system and a 707 scan head (VisualSonics Inc.) as described previously (38). The LV ejection fraction (EF) was calculated using the formula EF\% $=[(\mathrm{LVEDV}-\mathrm{LVESV}) / \mathrm{LVEDV}] \times 100$; where LVEDV and LVESV are LV end-diastolic volume and LV end-systolic volume, respectively.

Determination of infarct size. Myocardium was stained with 2\% TTC to measure infarct size as previously described (36). In brief, 72 hours after I/R, the slipknot around the LAD was retied, followed by injection of $2 \%$ Evans Blue dye $(0.2 \mathrm{ml})$. Hearts were excised, and the LV was sliced into three 1.2 -mm-thick slices perpendicular to the short axis of the heart and incubated in PBS containing TTC. After 20 minutes, at room temperature, the slices were digitally photographed. The Evans Blue-stained area (area not at risk), TTC-negative area (infarcted myocardium), and AAR (includes both TTC-negative and -positive areas) were measured with computer-based image analyzer SigmaScan Pro 5.0 (SPSS Science). AAR was expressed as percentage of total LV, while infarcted myocardium was expressed as percentage of AAR. For Western blot analysis, the border zone included the area of the ventricle $3 \mathrm{~mm}$ from the apex of the heart.

Statistics. Data were analyzed using Graph Pad Prizm 6 or JMP version 12. Data are presented as mean \pm SEM for continuous variables. Two-way ANOVA with Bonferroni multiple comparisons adjustments were used to assess differences across the investigational groups. A Student's 2-tailed $t$ test was used when there were only two groups for comparison. A $P$ value of $P<0.05$ was considered significant. The control for each experiment (e.g., Ad-GFP or normoxia) was set as 1.0 .

Study approval. All experiments were performed according to the National Institutes of Health Guide for the Care and Use of Laboratory Animals (National Academies Press. 2011) and were approved by the Temple University Institutional Animal Care and Use Committee (ACUP 4380) 


\section{Author contributions}

All authors read the manuscript and provided feedback. FS and AMF conceived the project. FS, JG, DGT, KK, JYC, and AMF designed the experiments. JYC and AMF supervised the project. FS and VDM performed the animal experiments, including echocardiography, and collected and analyzed the data. FS, JW, and TK performed the cell culture experiments and collected and analyzed the data. EG performed the animal surgery and, with FS, collected and analyzed the data. FGT, MKG, and JG provided input and help for the autophagy and apoptosis experiments. FS and MM performed the confocal microscopy and collected and analyzed the data. JR provided input regarding viral vectors and created the vectors used in the study. DGT provided input regarding the confocal microscopy and Western blot analysis as well as pathologic evaluations. KK provided advice and guidance about measures of autophagy and apoptosis and BAG3. FS and JW prepared the figures. FVR performed statistical analysis. FS, JYC, and AMF wrote the manuscript.

\section{Acknowledgments}

This study was supported by grants from the National Institutes of Health (P01 HL 091799-01 to AMF and R01 HL123093 to KK, JYC, and AMF). The authors thank Denise Tierney for preparation of the manuscript.

Address correspondence to: Arthur M. Feldman, Department of Medicine, Lewis Katz School of Medicine at Temple University, 3401 North Broad Street, Suite C902 Parkinson Pavilion, Philadelphia, Pennsylvania 19140, USA. Phone: 215.707.4036; E-mail: Arthur.Feldman@tuhs.temple.edu.

1. Behl C. Breaking BAG: The cochaperone BAG3 in health and disease. Trends Pharmacol Sci. 2016;37(8):672-688.

2. Knezevic T, et al. BAG3: a new player in the heart failure paradigm. Heart Fail Rev. 2015;20(4):423-434

3. Homma S, Iwasaki M, Shelton GD, Engvall E, Reed JC, Takayama S. BAG3 deficiency results in fulminant myopathy and early lethality. Am J Pathol. 2006;169(3):761-773.

4. Odgerel Z, et al. Inheritance patterns and phenotypic features of myofibrillar myopathy associated with a BAG3 mutation. Neuromuscul Disord. 2010;20(7):438-442.

5. Selcen D, et al. Mutation in BAG3 causes severe dominant childhood muscular dystrophy. Ann Neurol. 2009;65(1):83-89.

6. Feldman AM, et al. Decreased levels of BAG3 in a family with a rare variant and in idiopathic dilated cardiomyopathy. $J$ Cell Physiol. 2014;229(11):1697-1702.

7. Norton N, et al. Genome-wide studies of copy number variation and exome sequencing identify rare variants in BAG3 as a cause of dilated cardiomyopathy. Am J Hum Genet. 2011;88(3):273-282.

8. Hishiya A, Kitazawa T, Takayama S. BAG3 and Hsc70 interact with actin capping protein CapZ to maintain myofibrillar integrity under mechanical stress. Circ Res. 2010;107(10):1220-1231.

9. Feldman AM, et al. BAG3 regulates contractility and $\mathrm{Ca}(2+)$ homeostasis in adult mouse ventricular myocytes. J Mol Cell Cardiol. 2016;92:10-20.

10. Knezevic T, et al. Adeno-associated virus serotype 9 - driven expression of BAG3 significantly improves left ventricular function in murine hearts with left ventricular dysfunction secondary to a myocardial infarction. JACC Basic Transl Sci. In press.

11. Jackson S, Schaefer J, Meinhardt M, Reichmann H. Mitochondrial abnormalities in the myofibrillar myopathies. Eur J Neurol. 2015;22(11):1429-1435.

12. Tahrir FG, et al. Evidence for the role of BAG3 in mitochondrial quality control in cardiomyocytes [published online ahead of print July 6, 2016]. J Cell Physiol. doi: 10.1002/jcp.25476.

13. Gottlieb RA, Pourpirali S. Lost in translation: miRNAs and mRNAs in ischemic preconditioning and ischemia/reperfusion injury. J Mol Cell Cardiol. 2016;95:70-77.

14. Pell VR, Chouchani ET, Murphy MP, Brookes PS, Krieg T. Moving Forwards by Blocking Back-Flow: The Yin and Yang of MI Therapy. Circ Res. 2016;118(5):898-906.

15. Barth S, Glick D, Macleod KF. Autophagy: assays and artifacts. J Pathol. 2010;221(2):117-124.

16. Eskelinen EL, et al. Role of LAMP-2 in lysosome biogenesis and autophagy. Mol Biol Cell. 2002;13(9):3355-3368.

17. Huynh KK, Eskelinen EL, Scott CC, Malevanets A, Saftig P, Grinstein S. LAMP proteins are required for fusion of lysosomes with phagosomes. EMBO J. 2007;26(2):313-324.

18. Nishino I, et al. Primary LAMP-2 deficiency causes X-linked vacuolar cardiomyopathy and myopathy (Danon disease). Nature. 2000;406(6798):906-910.

19. Tanaka Y, et al. Accumulation of autophagic vacuoles and cardiomyopathy in LAMP-2-deficient mice. Nature. 2000;406(6798):902-906.

20. Yang Z, Klionsky DJ. Eaten alive: a history of macroautophagy. Nat Cell Biol. 2010;12(9):814-822.

21. Nuñez G, Benedict MA, Hu Y, Inohara N. Caspases: the proteases of the apoptotic pathway. Oncogene. 1998;17(25):3237-3245.

22. Slee EA, Adrain C, Martin SJ. Executioner caspase-3, -6, and -7 perform distinct, non-redundant roles during the demolition phase of apoptosis. J Biol Chem. 2001;276(10):7320-7326.

23. Klionsky DJ, et al. Guidelines for the use and interpretation of assays for monitoring autophagy. Autophagy. 2012;8(4):445-544

24. Ma X, et al. Impaired autophagosome clearance contributes to cardiomyocyte death in ischemia/reperfusion injury. Circulation. 2012;125(25):3170-3181.

25. Lavandero S, Troncoso R, Rothermel BA, Martinet W, Sadoshima J, Hill JA. Cardiovascular autophagy: concepts, controver- 
sies, and perspectives. Autophagy. 2013;9(10):1455-1466.

26. Gatica D, Chiong M, Lavandero S, Klionsky DJ. Molecular mechanisms of autophagy in the cardiovascular system. Circ Res. 2015;116(3):456-467.

27. Abbate A, et al. Increased myocardial apoptosis in patients with unfavorable left ventricular remodeling and early symptomatic post-infarction heart failure. J Am Coll Cardiol. 2003;41(5):753-760.

28. Wencker D, et al. A mechanistic role for cardiac myocyte apoptosis in heart failure. J Clin Invest. 2003;111(10):1497-1504

29. Kyriakis JM, Avruch J. Mammalian MAPK signal transduction pathways activated by stress and inflammation: a 10-year update. Physiol Rev. 2012;92(2):689-737.

30. Bogoyevitch MA, et al. Stimulation of the stress-activated mitogen-activated protein kinase subfamilies in perfused heart. p38/RK mitogen-activated protein kinases and c-Jun N-terminal kinases are activated by ischemia/reperfusion. Circ Res. 1996;79(2):162-173

31. Lei Z, Brizzee C, Johnson GV. BAG3 facilitates the clearance of endogenous tau in primary neurons. Neurobiol Aging. 2015;36(1):241-248.

32. Wang HQ, et al. Inhibition of the JNK signalling pathway enhances proteasome inhibitor-induced apoptosis of kidney cancer cells by suppression of BAG3 expression. Br J Pharmacol. 2009;158(5):1405-1412.

33. Li C, et al. BAG3 is upregulated by c-Jun and stabilizes JunD. Biochim Biophys Acta. 2013;1833(12):3346-3354.

34. Gentilella A, Khalili K. Autoregulation of co-chaperone BAG3 gene transcription. J Cell Biochem. 2009;108(5):1117-1124

35. Seok J, et al. Genomic responses in mouse models poorly mimic human inflammatory diseases. Proc Natl Acad Sci U S A. 2013;110(9):3507-3512.

36. Tao L, et al. Adiponectin cardioprotection after myocardial ischemia/reperfusion involves the reduction of oxidative/nitrative stress. Circulation. 2007;115(11):1408-1416

37. Zhu W, Tilley DG, Myers VD, Coleman RC, Feldman AM. Arginine vasopressin enhances cell survival via a G protein-coupled receptor kinase 2/ $\beta$-arrestin1/extracellular-regulated kinase 1/2-dependent pathway in H9c2 cells. Mol Pharmacol. 2013;84(2):227-235

38. Tilley DG, et al. $\beta$-adrenergic receptor-mediated cardiac contractility is inhibited via vasopressin type 1A-receptor-dependent signaling. Circulation. 2014;130(20):1800-1811.

39. Song J, et al. Regulation of cardiac myocyte contractility by phospholemman: $\mathrm{Na}+/ \mathrm{Ca} 2+$ exchange versus $\mathrm{Na}+-\mathrm{K}+-\mathrm{ATPase}$. Am J Physiol Heart Circ Physiol. 2008;295(4):H1615-H1625.

40. Zhou YY, et al. Culture and adenoviral infection of adult mouse cardiac myocytes: methods for cellular genetic physiology. Am J Physiol Heart Circ Physiol. 2000;279(1):H429-H436.

41. Tomar D, et al. MCUR1 Is a Scaffold Factor for the MCU Complex Function and Promotes Mitochondrial Bioenergetics. Cell Rep. 2016;15(8):1673-1685.

42. Castillo K, et al. Measurement of autophagy flux in the nervous system in vivo. Cell Death Dis. 2013;4:e917.

43. Li DL, et al. Doxorubicin Blocks Cardiomyocyte Autophagic Flux by Inhibiting Lysosome Acidification. Circulation. 2016;133(17):1668-1687.

44. Yardeni T, Eckhaus M, Morris HD, Huizing M, Hoogstraten-Miller S. Retro-orbital injections in mice. Lab Anim (NY) 2011;40(5):155-160. 